\title{
Universal macroscopic background formation in surface super-roughening
}

\author{
H.-W. Lee ${ }^{(1)}$ and Doochul Kim ${ }^{(2)}$ \\ ${ }^{(1)}$ Center for Theoretical Physics, Seoul National University, Seoul 151-742, Korea \\ ${ }^{(2)}$ Department of Physics and Center for Theoretical Physics, Seoul National University, Seoul 151-742, Korea
}

\begin{abstract}
We study a class of super-rough growth models whose structure factor satisfies the Family-Vicsek scaling. We demonstrate that a macroscopic background spontaneously develops in the local surface profile, which dominates the scaling of the local surface width and the height-difference. The shape of the macroscopic background takes a form of a finite-order polynomial whose order is decided from the value of the global roughness exponent. Once the macroscopic background is subtracted, the width of the resulting local surface profile satisfies the Family-Vicsek scaling. We show that this feature is universal to all super-rough growth models, and we also discuss the difference between the macroscopic background formation and the pattern formation in other models.
\end{abstract}

PACS number(s): 05.40.+j, 05.70.Ln, 68.35.Fx

Recently, there has been considerable progress in the understanding of the dynamics of growing surfaces [1.2]. Much of the progress is motivated by the crucial observation that surface roughening exhibits scaling behaviors. For example, the growth of the global surface width $w(L, t)$, when it starts from the flat surface, shows the following behavior. At an initial stage, $w(L, t)$ grows as a power law of time $w(L, t) \sim t^{\beta}$, where $\beta$ is called the growth exponent. At a later stage, however, $w(L, t)$ saturates to a certain power of the system size $L, w(L, t) \sim L^{\alpha}$, where $\alpha$ is called the roughness exponent. The crossover to the saturated surface is governed by the lateral correlation length $\xi(t)$, which scales as $t^{1 / z}$ for the initial stage $t \ll L^{z}$ and saturates to $L$ for the later stage $t \gg L^{z}$. Here the third exponent $z$ is called the dynamic exponent, and this scaling behavior is called the Family-Vicsek (FV) scaling ansatz [3].

The presence of the scaling is usually related to the self-affine structure of a surface, implying the same properties at all length scales. In some growth models (for example, Refs. [田:5]), however, surface properties exhibit different behaviors when they are probed at different length scales. For example, one may probe the surface width $w(l, t)$ within a local window of size $l(\ll L)$, where

$$
w^{2}(l, t)=\left\{\left\langle\left(h(x, t)-\langle h(x, t)\rangle_{X_{l}}\right)^{2}\right\rangle_{X_{l}}\right\},
$$

and \langle\rangle$_{X_{l}}$ is the spatial average over the local window $X_{l}$ of size $l$ and \{\} the sample average. And one finds

$$
w^{2}(l, t) \propto \begin{cases}t^{2 \beta} & \text { for } t \ll l^{z} \\ l^{2 \alpha}\left(\frac{\xi(t)}{l}\right)^{2\left(\alpha-\alpha^{\prime}\right)} & \text { for } t \gg l^{z}\end{cases}
$$

Note that while it follows the FV scaling at the initial stage $t \ll l^{z}$, the scaling behavior at the later stage is anomalous : the window size dependence is described not by the global roughness exponent $\alpha$, but rather by a new exponent $\alpha^{\prime}(<\alpha)$ which is called local roughness exponent. Note also that at the later stage, the surface width implied by Eq. (2) is anomalously larger than the FV scaling result $l^{2 \alpha}$, since $\alpha>\alpha^{\prime}$ and $\xi(t) \gg l$.

In the past few years, there have been many works on the anomalous roughening. Numerical simulations demonstrated the occurrence of the anomalous roughening in some growth models (for example, see Refs. (4.5)). New scaling ansatz for the anomalous roughening was proposed [6.7]. Very recently, López et al. [8] identified two separate mechanisms of the anomalous roughening, super-roughening and intrinsic roughening. In case of the super-roughening $(\alpha>1)$, the structure factor $S(k)$, or the power spectrum, follows the Family-Vicsek scaling and the anomalous roughening occurs due to the divergence of $S(k)$ near $k=0$. In case of the intrinsic anomalous roughening, on the other hand, $S(k)$ itself scales anomalously.

Among the two mechanisms, we focus in this paper on the anomalous roughening due to the super-roughening. In particular, we study the morphology of the local surface profile generated in the super-rough growth models. Usually, the surface profile can be probed from the scaling of the local surface width or the height-difference as a function of a probing length scale. In super-rough surfaces, however, the scaling is much less informative, since the relevant local roughness exponent $\alpha^{\prime}$ is always 1 [8] independent of many details of growth models, the only implication of the universal value 1 being the divergence of $S(k)$. So to probe the surface profile in the super-rough growth models, we take a different theoretical approach based on the least-square-fitting method. As a main result of the paper, we find that (i) the local surface profile forms a macroscopic background that takes the form of a finite order polynomial and that (ii) the magnitude of the short wavelength fluctuations superimposed on the macroscopic background follows the FV scaling. We also show that this property is universal to all super-rough growth models. 
One simple way to study the profile is to make a guess on the functional form of the profile, fit the function to the profile through the least-square-fitting method, and examine the magnitude of the discrepancy. The simplest example of this approach is the local surface width since the idea of Eq. (11) is nothing but the least-square-fitting of the local surface profile with a constant function $\langle h(x, t)\rangle_{X_{l}}$. Adopting this view point, it is then interesting to explore other possibilities of fitting functions, which hopefully produces a smaller discrepancy. Since the origin of the anomalous roughening in the super-rough growth models is the divergence in the long wavelength components that appear smooth in the local window, we first take a first-order polynomial in $x$ as a fitting function : $\tilde{h}_{1}(x, t)=a_{1}(t) x+a_{0}(t)$. Here, the coefficients $a_{i}(t)$ are fixed by minimizing the discrepancy $\left\langle\left[h(x, t)-\tilde{h}_{1}(x, t)\right]^{2}\right\rangle_{X_{l}}$ for a given surface height profile $h(x, t)$.

For illustration, we first use the $1+1$ dimensional uniform diffusion models as examples :

$$
\frac{\partial h(x, t)}{\partial t}=(-1)^{m+1} \frac{\partial^{2 m} h(x, t)}{\partial x^{2 m}}+\eta(x, t),
$$

where $\eta(x, t)$ is the noise with correlation $\left\{\eta(x, t) \eta\left(x^{\prime}, t^{\prime}\right)\right\}=D \delta\left(x-x^{\prime}\right) \delta\left(t-t^{\prime}\right)$, and $m=1,2,3, \ldots$. For an arbitrary $m$, it can be explicitly verified that the structure factor follows the FV scaling. Also the precise values of the scaling exponents are known : $\alpha=(2 m-1) / 2, \beta=(2 m-1) / 4 m$, and $z=2 m$ [9]. Notice that for $m \geq 2$, the uniform diffusion models become super-rough $(\alpha>1)$. Then as demonstrated in Ref. [8], the models exhibit the anomalous scaling with $\alpha^{\prime}=1$ for $m \geq 2$.

The accuracy of the fitting with the first-order polynomial can be estimated from the ensemble averaged minimal discrepancy,

$$
w_{1}^{2}(l, t)=\left\{\left\langle\left(h(x, t)-\tilde{h_{1}}(x, t)\right)^{2}\right\rangle_{X_{l}}\right\},
$$

where we call $w_{1}(l, t)$ first-order generalized surface width. For the linear models Eq. (3), $w_{1}(l, t)$ can be evaluated analytically in a straightforward way. At the initial stage $\xi(t) \ll l, w_{1}^{2}(l, t)$ scales the same way as $w^{2}(l, t)$, implying that there is no substantial improvement of the fitting from the new choice of the fitting function. This result is understandable since the divergence of $S(k)$ does not qualitatively affect the surface profile at the initial stage. At the later stage $\xi(t) \gg l$, however, the results are

$$
w_{1}^{2}(l, t) \propto \begin{cases}l^{2 \alpha} & \text { for } \alpha<2(\text { or } m=1,2) \\ l^{2 \alpha}\left(\frac{\xi(t)}{l}\right)^{2(\alpha-2)} & \text { for } \alpha>2(\text { or } m \geq 3) .\end{cases}
$$

Here the reduction in the power of the large factor $\xi(t) / l$ should be noticed : it has been reduced from $2(\alpha-1)$ in $w^{2}(l, t)$ to 0 for $m=2$ and to $2(\alpha-2)$ for $m \geq 3$. For the definiteness, let us take $l / \xi(t)$ as a small parameter of the analysis. Then the power reduction implies the substantial improvement of the fitting, which we interpret as an evidence of a spontaneous formation of a macroscopic background in the surface profile. Here the word "macroscopic" denotes that the characteristic length scale of the background is comparable to or larger than the window size $l$. Figure 1 shows a typical surface profile of the $m=2$ uniform diffusion model at the later stage $\xi(t) \gg l$, and the formation of the linear background is clear 10].

Motivated by the success of the first-order polynomial, we explore the idea further and examine the other types of fitting functions. Specifically we choose a higher-order polynomial, which is a natural extension of the first-order polynomial. One merit of this choice is that polynomials form a complete set of basis for functional space. So by examining the fitting with high-order polynomials, one can examine the effect of all types of fitting functions. To estimate the accuracy of the fitting with an $N$ th order polynomial, we introduce an $N$ th order generalized surface width $w_{N}(l, t)$, where

$$
w_{N}^{2}(l, t)=\left\{\left\langle\left[h(x, t)-\tilde{h_{N}}(x, t)\right]^{2}\right\rangle_{X_{l}}\right\}
$$

and the coefficients $a_{i}(t)$ of $\tilde{h}_{N}(x, t)=\sum_{i=0}^{N} a_{i}(t) x^{i}$ are similarly fixed by the least-square-fitting method for a given realization $h(x, t)$. For the linear model Eq. (3), one can analytically verify that $w_{N}^{2}(l, t)$ scales the same way as $w^{2}(l, t)$ at the initial stage, again finding no improvement, and

$$
w_{N}^{2}(l, t) \propto\left\{\begin{array}{ll}
l^{2 \alpha} & \text { for } \alpha<N+1(\text { or } m \leq N+1) \\
l^{2 \alpha}\left(\frac{\xi(t)}{l}\right)^{2(\alpha-N-1)} & \text { for } \alpha>N+1(\text { or } m \geq N+2)
\end{array},\right.
$$


at the later stage. This scaling result contains very interesting information. First, the accuracy improves substantially as the order $N$ increases till $N$ becomes larger than $\alpha-1$, after which it saturates and does not improve upon the further increase of $N$. Recalling the completeness of the polynomial basis, one then realizes that even if other forms of fitting function were chosen, such as sines and cosines, they would not improve the accuracy either. In this sense, one can say that the macroscopic background in the uniform diffusion models takes the form of an $N$ th order polynomial, where $N$ is the largest integer smaller than $\alpha$. Second, the minimal discrepancy of the fitting is always of the order of $l^{2 \alpha}[1]$, and so it is characterized by the global roughness exponent. One interesting consequence of this result is that once the macroscopic background is subtracted, the width of the resulting local surface profile satisfies the FV scaling.

To understand the underlying physics in the uniform diffusion models, let us represent the local surface profile in the following way : $h(x, t)=\sum_{i=0}^{2 m-1} a_{i}(t) x^{i}+\sum_{k}[A(k, t) \sin k x+B(k, t) \cos k x]$, where $k l$ is an integer multiple of $2 \pi$. One interesting property of the uniform diffusion models is that while the relaxation force tends to suppress the amplitude of the sinusoidal part, it does not affect the polynomial part : $F\left[\sum_{i=0}^{2 m-1} a_{i}(t) x^{i}\right]=0$ for arbitrary $a_{i}(t)$ where $F[h(x, t)]=(-1)^{m+1} \partial^{2 m} h / \partial x^{2 m}$. This property opens a possibility for the anomalous growth of the coefficients $a_{i}(t)$, which makes polynomials special candidates for a fitting function. And the scaling result of $w_{N}^{2}(l, t)$ implies that the coefficients $a_{i}(t)$ with $i<\alpha$ (or $i<m$ ) indeed become anomalously large so that they lead to the formation of the macroscopic background.

Let us compare the formation of the macroscopic background in the uniform diffusion models with pattern formation in other models where the pattern formation is due to symmetry breaking or the coherent superposition of modes of different wavelength [12]. In the uniform diffusion models, the symmetry breaking does not occur and the ensemble average of $a_{i}(t)$ vanishes. Also there is no coherent coupling between different modes since the models are linear. Here, however, the fluctuations of $a_{i}(t)$ in different realizations are so large that the fluctuations lead to the macroscopic background formation for any given realization, that is, the macroscopic background formation is due to large fluctuations in some particular "degrees of freedom". In this sense, the macroscopic background formation is completely different from the pattern formation in its origin.

For the $1+1$ dimensional uniform diffusion models, it is now demonstrated that (i) a macroscopic background develops spontaneously in the local surface profile, and the background takes a form of an $N$ th order polynomial where $N$ is the largest integer smaller than $\alpha$, and that (ii) once the macroscopic background is subtracted, the resulting local surface profile satisfies the FV scaling. Below, we show that these properties are universal to all super-rough growth models.

From now on, we restrict the discussion to the later stage since it is trivial to show that $w_{N}^{2}(l, t)$ satisfies the FV scaling at the initial stage for an arbitrary $N$. To study the scaling behavior at the later stage, it is useful to first relate the height-height correlation function $G(x, t)$ to the structure factor $S(k, t)=\{\hat{h}(k, t) \hat{h}(-k, t)\}$. By comparing the definitions, one can obtain the following relation between $G(x, t)$ and $S(k, t)$ [8] :

$$
G(x, t)=4 \int_{k_{0}}^{\infty} \frac{d k}{2 \pi}[1-\cos (k x)] S(k, t),
$$

where the lower cutoff $k_{0}$ of the integration is decided by the system size $L, k_{0}=2 \pi / L$. Usually the lower cutoff can be replaced by 0 and Eq. (8) leads to $G(x, t)$ satisfying the $\mathrm{FV}$ scaling. For $\alpha>1$, however, the integration has the infrared divergence and the lower cutoff becomes important. In this case, by taking explicit care of the lower cutoff and from the FV scaling of $S(k, t)$ [for the FV scaling of $S(k, t)$, see, for example, Ref. [2]], one can obtain for $\xi(t) \gg x$,

$$
G(x, t)=|x|^{2 \alpha} u\left(x / t^{1 / z}\right)+|x|^{2} \xi^{2(\alpha-1)}(t) v(x / \xi(t))
$$

where $u(y \rightarrow 0)=u_{0}\left[u_{0} \log (1 / y)\right.$ if $\alpha$ is an integer $]$, and $v(y)$ is an even function of $y, v(y)=\sum_{i=0}^{\infty} v_{2 i} y^{2 i}$. In Eq. (9), the first term is the usual Family-Vicsek contribution and the second is the anomalous contribution coming from the integration near the lower cutoff. The even form of the scaling function $v(y)$ originates from the series expansion of the only $x$-dependent factor $1-\cos (k x)$ in Eq. (8). Notice that the anomalous contribution is dominant over the FV contribution for $\alpha>1$.

To demonstrate the universality, we show that the result in Eq. (7), regarding the scaling of $w_{N}^{2}(l, t)$, comes naturally from the form of $G(x, t)$ in Eq. (9). For simplicity, let us take $N=1$. The generalization to a larger value of $N$ is also straightforward and it will be sketched below. The key element of the demonstration is to derive a general relation between $w_{1}^{2}(l, t)$ and $G(x, t)$. From the definition of $w_{1}^{2}(l, t)$, one can verify the following linear relation :

$$
w_{1}^{2}(l, t)=\frac{1}{2 l^{2}} \int_{X_{l}} d x_{1} d x_{2} G\left(x_{1}-x_{2}, t\right)\left(1+\frac{12}{l^{2}} x_{1} x_{2}\right),
$$


where the center of the local window $X_{l}$ is chosen as the origin of the coordinate system. Then by combining Eq. (10) with Eq. (9), one finds

$$
w_{1}^{2}(l, t)=\sum_{i=0}^{\infty} f_{1}(i+1) v_{2 i} l^{2 \alpha}\left(\frac{\xi(t)}{l}\right)^{2(\alpha-1-i)}+f_{1}(\alpha) u_{0} l^{2 \alpha}+\cdots
$$

where

$$
f_{1}(\mu)=\frac{4(1-\mu)}{(2 \mu+1)(2 \mu+2)(2 \mu+4)},
$$

and the dots represent other terms smaller than $l^{2 \alpha}$. Then to find out the scaling behavior of $w_{1}^{2}(l, t)$, one just needs to select the leading order term from Eq. (11). In power counting of $l / \xi(t), f_{1}(1) v_{0} l^{2} \xi(t)^{2(\alpha-1)}$ appears to be the leading order term. However this term is not the true leading order term, since the proportionality constant $f_{1}(1)$ vanishes identically. Then by choosing the next leading order term in power counting, one can show that Eq. (5), which is a special case of Eq. (7), is universal.

The generalization of the analysis to a larger value of $N$ can be carried out in a similar way. From the definition of $w_{N}^{2}(l, t)$, one can derive a linear relation between $w_{N}^{2}(l, t)$ and $G(x, t)$. By combining this relation with Eq. (9), one finds

$$
w_{N}^{2}(l, t)=\sum_{i=0}^{\infty} f_{N}(i+1) v_{2 i} l^{2 \alpha}\left(\frac{\xi(t)}{l}\right)^{2(\alpha-1-i)}+f_{N}(\alpha) u_{0} l^{2 \alpha}+\cdots
$$

where the dots represent other terms smaller than $l^{2 \alpha}$, and $f_{N}(\mu)$ is zero if $\mu$ is an integer smaller than or equal to $N$ [13]. Then by selecting the true (nonvanishing) leading order term from Eq. (13), one can show the universality of Eq. (7)

So far, we have demonstrated the universality of the macroscopic background formation only for $1+1$ dimensional systems. However the universality is not restricted to $1+1$ dimensional systems. For illustration, here we show the universality of Eq. (5) for $D+1$ dimensional systems. The generalization of the two key relations Eqs. (8, 10) can be achieved through trivial replacements such as $k x \rightarrow \mathbf{k} \cdot \mathbf{x}$ and $x_{1} x_{2} \rightarrow \mathbf{x}_{\mathbf{1}} \cdot \mathbf{x}_{\mathbf{2}}$, where a $D$-dimensional cube with volume $l^{D}$ is chosen as a $D$-dimensional local window. Then following the same procedure, one obtains precisely the same expression as Eq. (11) except for a replacement of $f_{1}(\mu)$ by $f_{1, D}(\mu)=D f_{1}(\mu)$, and one verifies the universality in higher spatial dimensions. The generalization of the analysis to a larger value of $N$ is also straightforward.

One important signature of the super-rough growth models is the universal value of the local roughness exponent $\alpha^{\prime}=1$. For some growth models [5, 14, 15], numerical simulations resulted in $\alpha^{\prime} \approx 1$, and so it would be interesting to examine whether the present analysis applies to those growth models. One of the most promising candidates is the $1+1$ dimensional model of driven interfaces in random media, for which the functional renormalization group study by Narayan and Fisher [16] resulted in $\alpha^{\prime}=1$ in the critical region.

In this paper, we consider the anomalous roughening in super-rough $(\alpha>1)$ growth models whose structure factors satisfy the Family-Vicsek scaling. We show that these growth models share the following universal features. First, the local surface profile is characterized by a formation of a macroscopic background, which takes a form of an $N$ th order polynomial, where $N$ is the largest integer smaller than the global exponent $\alpha$. Second, after subtracting the macroscopic background, the width of the resulting local surface profile satisfies the Family-Vicsek scaling.

We would like to acknowledge helpful discussions with J. M. Kim. This work is supported by the Korea Science and Engineering Foundation through the SRC program of SNU-CTP.

[1] Dynamics of Fractal Surfaces, edited by F. Family and T. Vicsek (World Scientific, Singapore, 1991), Chap. 3, p. 73.

[2] A.-L. Barabási and H. E. Stanley, in Fractal Concepts in Surface Growth (Cambridge University Press, Cambridge, 1995).

[3] F. Family and T. Vicsek, J. Phys. A 18, L75 (1985).

[4] J. G. Amar, P.-M. Lam, and F. Family, Phys. Rev. E 47, 3242 (1993).

[5] L. A. N. Amaral, A. L. Barabasi, H. A. Makse, and H. E. Stanley, Phys. Rev. E 52, 4087 (1995). 
[6] M. Schroeder, M. Siegert, D. E. Wolf, J. D. Shore, and M. Plischke, Europhys. Lett. 24, 563 (1993).

[7] S. Das Sarma, S. V. Ghaisas, and J. M. Kim, Phys. Rev. E 49, 122 (1994).

[8] J. M. López, M. A. Rodríguez, and R. Cuerno, Report No. cond-mat/9703024.

[9] J. M. López and M. A. Rodríguez, Phys. Rev. E 54, R2189 (1996).

[10] The formation of the linear background has been previously identified by Amar et al. 此 as an origin of the anomalous roughening for the $m=2$ uniform diffusion model, which they call groove instability.

[11] The proportionality constant in front of the minimal discrepancy $l^{2 \alpha}$ decays as $N \rightarrow \infty$. However, this decay does not change the scaling of the minimal discrepancy since the relevant limiting procedure is to take $\xi(t) / l \rightarrow \infty$ first and then allow $N \rightarrow \infty$.

[12] For example, see M. Lücke, M. Mihelcic, B. Kowalski, and K. Wingerach, in The Physics of Structure Formation, edited by W. Güttinger and G. Dangelmayr (Springer-Verlag, Berlin, 1987).

[13] For an integer $\alpha$, the FV term $f_{N}(\alpha) u_{0} l^{2 \alpha}$ in Eq. (13) is modified to $f_{N}^{\prime}(\alpha) u_{0} l^{2 \alpha} \log \left[\kappa_{N}(\alpha) \xi(t) / l\right]$ where $\kappa_{N}(\alpha)$ is a constant of order 1. The modification occurs because the $y \rightarrow 0$ limiting behavior of the scaling function $u(y)$ [Eq. (9)] is modified from $u_{0}$ to $u_{0} \log (1 / y)$ for an integer $\alpha$. The new proportionality constant $f_{N}^{\prime}(\alpha)$ is not zero and so the FV term survives even if $\alpha$ is an integer.

[14] H. J. Jensen, J. Phys. A 28, 1861 (1995).

[15] M. Dong, M. C. Marchetti, A. A. Middleton, and V. Vinokur, Phys. Rev. Lett. 70, 662 (1993).

[16] O. Narayan and D. S. Fisher, Phys. Rev. B 48, 7030 (1993).

FIG. 1. Typical surface profile of a $1+1$ dimensional uniform diffusion model with $m=2$ [Eq. (3)] at the later stage $\xi(t) \gg l$. Notice the formation of a linear macroscopic background $\tilde{h}_{1}(x, t)$ whose large slope leads to the anomalous scaling of $w^{2}(l, t)$. Once the background is subtracted, the resulting local surface width $w_{1}(l, t)$ is characterized by the global roughness exponent $\alpha$ and satisfies the Family-Vicsek scaling. 
Fig. 1

$h(x, t)$

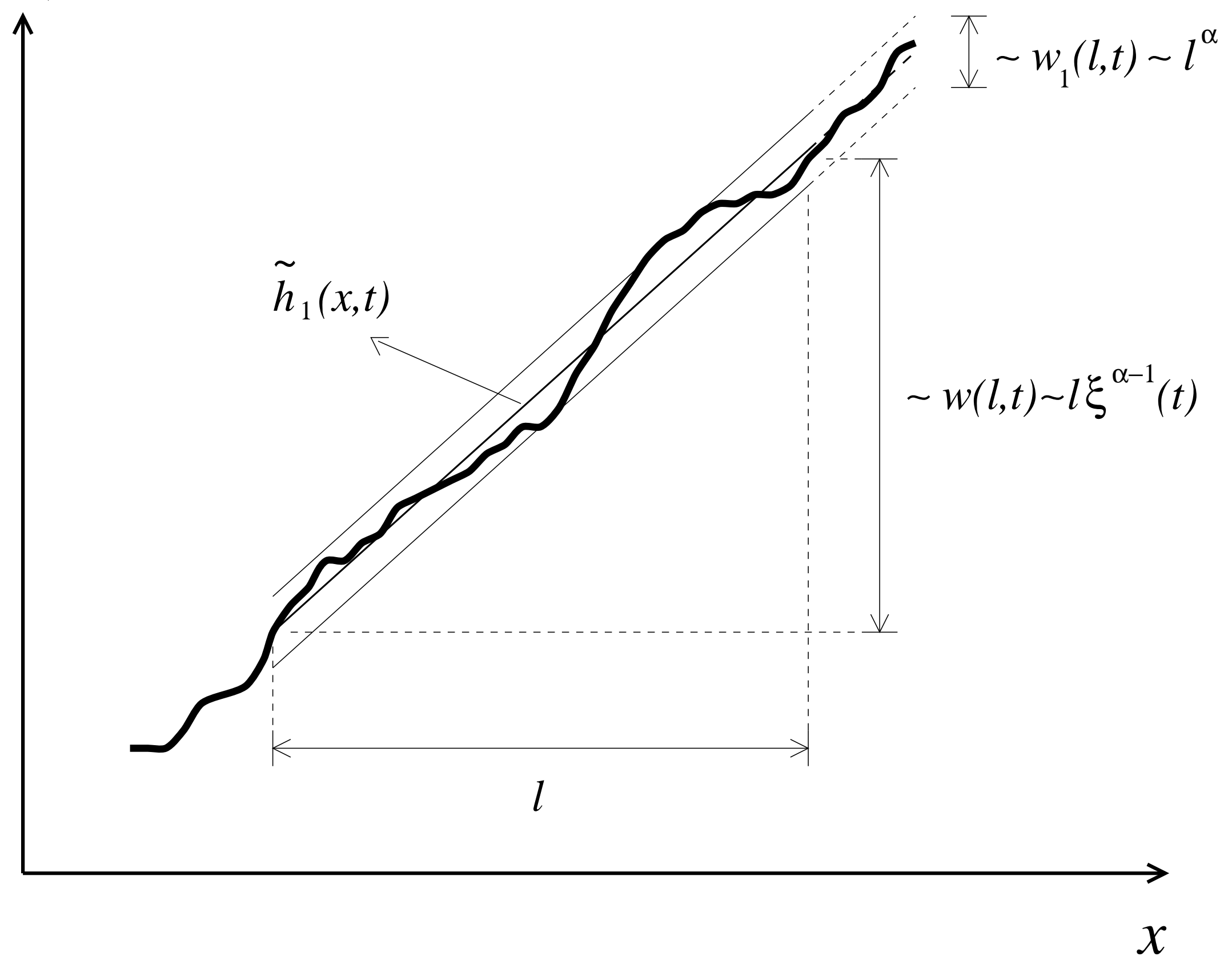

